\title{
The Role of Exercise Physical Activity in Varying the Total Energy Expenditure in Healthy Japanese Men 30 to 69 Years of Age
}

\author{
Hoby Hasina RafamantanAnTSOA ${ }^{1}$, Naoyuki Ebine ${ }^{2}$, Mayumi Yoshioka ${ }^{3}$, Yutaka Yoshitake ${ }^{4}$, \\ Hiroaki TANAKA ${ }^{3}$, Shinichi SAITOH ${ }^{1, *}$ and Peter John Harris JoNES ${ }^{2}$ \\ ${ }^{1}$ Institute of Health and Sport Sciences, University of Tsukuba, Tennoudai 1-1-1, Tsukuba, 305-8574, Japan \\ ${ }^{2}$ School of Dietetics and Human Nutrition, McGill University, Quebec H9X 3V9, Canada \\ ${ }^{3}$ Department of Health and Sports Sciences, Fukuoka University, \\ Nanakuma 8-19-1, Jonan-ku, Fukuoka 814-0180, Japan \\ ${ }^{4}$ National Institute of Fitness and Sports in Kanoya, \\ Shiromizu 1-1, Kanoya, Kagoshima 891-2393, Japan
}

(Received September 18, 2002)

\begin{abstract}
Summary This study was designed to examine 1) the role of exercise physical activity (EPA), and then 2) physical fitness and body composition upon variation of the total energy expenditure (TEE) in healthy Japanese men aged 30 to $69 \mathrm{y}(n=40)$. EPA and TEE were assessed over $14 \mathrm{~d}$ using an accelerometer and a doubly labeled water (DLW) method, respectively. Basal metabolic rate (BMR) was measured after $10 \mathrm{~h}$ fasting on the morning of the day of DLW dosing. Physical activity-induced energy expenditure (PAEE) was calculated by subtracting BMR and diet-induced thermogenesis (DIT $=10 \%$ TEE) from TEE. EPA was subdivided into three intensities: low, moderate and high and the accumulated duration (time expressed in minutes) of each of these was calculated. Body composition and physical fitness $\left(\dot{\mathrm{V}} \mathrm{O}_{2} \max \right)$ were determined using an underwater weighing method and a treadmill exercise test, respectively. BMR (mean \pm SD: 1,459 $\pm 181 \mathrm{kcal} / \mathrm{d}$ ) declined significantly with age $(r=-0.37, p<0.05)$, but PAEE $(946 \pm 320 \mathrm{kcal} / \mathrm{d})$ and TEE $(2,672 \pm 369 \mathrm{kcal} / \mathrm{d}) \mathrm{did}$ not. A multiple stepwise regression analysis was used to develop an empirical model that relates energy expenditure measured by the DLW (TEE) to age, height, body mass index, FM, FFM, percentage body fat, $\dot{\mathrm{V}} \mathrm{O}_{2}$ max, and accumulated duration spent for low-, moderate-, and high-intensity EPA. The results revealed that FFM and high-intensity EPA were identified as important determinants of TEE and accounted for $51 \%$. We may therefore conclude that 1 ) high-intensity EPA appears to be relevant in determining TEE, especially among active individuals, and 2) body composition was more important than physical fitness in determining TEE in this population.
\end{abstract}

Key Words total energy expenditure, doubly labeled water method, exercise physical activity, accelerometer, age 30 to $69 \mathrm{y}$-old

Since the mid-20th century, there has been growing scientific information on the health benefits of being physically active $(1,2)$. Today, physical activity has a central role in health system and social efforts that are directed towards the prevention of daily risk-related disease outcomes. Thus, the assessment of physical activity level (PAL) and measurement of total energy expenditure (TEE) in free-living individuals under a variety of circumstances has become an area of particular interest $(3,4)$. It is well recognized that physical inactivity may be associated with both a decrease in physical fitness and daily energy expenditure, and hereby a decrease in the latter with age greatly increases the chance of becoming overweight (5). This most recent shift in focus has resulted in the need for a more precise quantification of activity level and energy expenditure in free-living peoples $(6,7)$.

* To whom correspondence should be addressed.

E-mail: ssp@taiiku.tsukuba.ac.jp
As the apparent benefits associated with an active lifestyle can be gauged by examining habitual physical activity or energy expenditure per se, ensuing healthrelated markers such as loss of body fat or improvement of physical fitness $\left(\dot{\mathrm{V}} \mathrm{O}_{2}\right.$ max $)$ are of interest. Using whole room indirect calorimetry, Melanson et al. recently revealed that, as compared to sedentary subjects, individuals following either a low- or high-intensity exercise program had a higher TEE. However, there was found to be no difference in TEE between the two exercise groups (8). Westerterp, using both an accelerometer and the DLW method, found that the accumulated duration of moderate-intensity physical activity appeared to be more effective in increasing PAL than low-intensity exercise in the middle-aged (5). In the same study, the total proportion of high-intensity exercise did not appear to influence TEE. However, it has also been recognized that high-intensity exercise can have a potential impact on energy expenditure in a number of ways: first, exercise-associated energy expenditure is in- 
creased by increasing work rate; second, resistance training increases muscle mass and in turn increases resting energy expenditure; and third, participating in aerobic exercise at an intensity greater than $70 \%$ $\dot{\mathrm{V}} \mathrm{O}_{2}$ max has been found to increase resting energy expenditure without any change in muscle mass (9). Although exercise physical activity (EPA) is therefore believed to play a role in the beneficial effects of healthrelated fitness, the relationship between its quantification and TEE is not yet fully known. In addition the doubly labeled water (DLW) method has been adopted as a gold standard to assess human total energy expenditure (TEE) under field conditions (10), but there is no such gold standard for measuring physical activity. The development of several methods for assessing physical activity has not yet solved the ongoing problem of deficit in between study accuracy and feasibility.

In a previous study of young Japanese men, Ebine et al. reported that using an accelerometer represents a promising technique for measuring free-living TEE as compared to activity records and heart rate monitoring (11). In a recent study of older Japanese men, we also found that an accelerometer was a useful and effective device that allowed us to differentiate the duration and intensity of daily physical activity (12).

Thus, the present study was conducted using an accelerometer to examine the role of EPA (especially its duration and intensity) in effecting TEE in healthy Japanese men 30 to $69 \mathrm{y}$ of age. In addition, the contribution of body composition and physical fitness in determining TEE was also investigated.

\section{METHODS}

Subjects. Forty healthy, working Japanese male volunteers, representing four age categories from 30 to 39 , 40 to 49,50 to 59 , and 60 to $69 \mathrm{y}$ ( $n=10$ per group), participated in the study. They are civil servants (e.g. teachers, librarians, medical doctors, nurses, office workers, drivers), recruited from Fukuoka City and the surrounding area. Informed consent was obtained from all participants prior to the investigations. Anthropometric measures are presented in Table 1. Volunteer thyroid status (triiodothyronine, thyroxine, and thyrotropin) was screened and found to fall within the normal range. Over the assessment period, subjects were instructed to maintain their daily lifestyle. This study was approved by the ethical committee of the National Institute of Health and Nutrition in Japan.

Experimental design. TEE was measured using the DLW method over a 14-d period. A LifeCorder accelerometer (AC) was worn by all subjects over the same period at all times while awake to gauge habitual physical activity. Measurements of subjects' physical characteristics, body composition, and basal metabolic rate (BMR), followed by the administration of a single oral dose of DLW $\left({ }^{2} \mathrm{H}_{2}{ }^{18} \mathrm{O}\right)$ were performed one day prior to the 14-d period of TEE measurement (day 0). Urinary samples were collected at $3 \mathrm{~h}$ and $4 \mathrm{~h}$ after ingestion to determine dilution space, and on day 1 and day 15 for isotopic elimination analysis of ${ }^{2} \mathrm{H}$ and ${ }^{18} \mathrm{O}$. A treadmill
Table 1. Physical characteristics, body composition, energy expenditure, and PAL of subjects $(n=40)$.

\begin{tabular}{lcc}
\hline & Mean \pm SD & Range \\
\hline Age $(\mathrm{y})$ & $50 \pm 12$ & $30-69$ \\
Body weight $(\mathrm{kg})$ & $65.5 \pm 10.1$ & $49.3-89.6$ \\
Height $(\mathrm{cm})$ & $167.3 \pm 6.4$ & $155.0-189.0$ \\
BMI $\left(\mathrm{kg} / \mathrm{m}^{2}\right)$ & $23.1 \pm 2.6$ & $19.6-29.5$ \\
Body fat $(\%)$ & $20.1 \pm 6.1$ & $10.3-32.6$ \\
Fat-free mass $(\mathrm{kg})$ & $52.2 \pm 8.5$ & $37.6-73.2$ \\
Fat mass $(\mathrm{kg})$ & $13.3 \pm 4.9$ & $5.9-26.9$ \\
$\dot{\mathrm{VO}} \mathrm{O}_{2} \mathrm{max}(\mathrm{mL} / \mathrm{kg} / \mathrm{min})$ & $38.8 \pm 8.4$ & $16.9-53.3$ \\
$\mathrm{TEE}(\mathrm{kcal} / \mathrm{d})$ & $2,672 \pm 369$ & $2,045-3,769$ \\
$\mathrm{BMR}(\mathrm{kcal} / \mathrm{d})$ & $1,459 \pm 181$ & $1,169-1,835$ \\
$\mathrm{PAEE}(\mathrm{kcal} / \mathrm{d})$ & $946 \pm 320$ & $341-1,905$ \\
$\mathrm{PAL}$ & $1.85 \pm 0.28$ & $1.35-2.53$ \\
\hline
\end{tabular}

BMI: body mass index, TEE: total energy expenditure, BMR: basal metabolic rate, PAEE: physical activity-induced energy expenditure, PAL: physical activity level.

exercise test was performed after collection of the last urinary sample.

Measurements.

Anthropometry and body composition: Body composition was measured using an underwater weighing method based on Brozek's description (13). The results are shown in Table 1. Body mass index (BMI) was calculated by dividing a subject's body weight in kilograms by the square of his height in meters $\left(\mathrm{kg} / \mathrm{m}^{2}\right)$.

Basal metabolic rate: Subjects fasted for $10 \mathrm{~h}$ prior to the beginning of test procedures on the morning of day 0 . Participants rested in a supine position for $30 \mathrm{~min}$ to collect exhaled air, and then the expired air was analyzed using a ventilated hood and an ARCO-1000 respiratory mass spectrometer (ARCO System Inc., Japan) for oxygen consumption and carbon dioxide production. Energy expenditure during rest was then calculated using Weir's equation (14).

Total energy expenditure: After collection of a urinary sample for a baseline measurement (day 0), each subject ingested a dose of approximately $0.12 \mathrm{~g} / \mathrm{kg}$ estimated total body water (eTBW) of $2 \mathrm{H}_{2} \mathrm{O}(99.8$ atom\%) and $0.25 \mathrm{~g} / \mathrm{kg}$ eTBW of $\mathrm{H}_{2}{ }^{18} \mathrm{O}$ (10.0 atom $\left.\%\right)$. eTBW was estimated as being $60 \%$ of each individual's body weight (15). Subjects refrained from eating or drinking for a $4 \mathrm{~h}$ equilibration period (urinary samples were collected at 3 and $4 \mathrm{~h}$ after the dose administration). Then, in the mornings on day 1 and day 15 , second void samples were collected for isotope elimination analysis. ${ }^{2} \mathrm{H}$ enrichment was analyzed using a 903D dual-inlet isotope ratio mass spectrometer (IRMS, VG Isogas, Cheshire, UK) calibrated using Vienna Standard Mean Ocean Water (V-SMOW), 302B, and Greenland Ice Sheet Precipitation Standard (GISP). ${ }^{18} \mathrm{O}$ enrichment was determined using a SIRA 12 IRMS (VG Isogas, Cheshire, UK). Analyses were performed in triplicate. The average standard deviation through the analysis was $1.77 \%$ o for ${ }^{2} \mathrm{H}$ and $0.20 \%$ of for ${ }^{18} \mathrm{O}$. Details of the method were assessed as previously described (16). TEE 
was expressed as the mean TEE over the 14-d assessment period.

Physical activity-induced energy expenditure (PAEE) was calculated by subtracting BMR and diet-induced thermogenesis (DIT $=10 \%$ TEE) from TEE. Physical activity level (PAL) was also calculated (PAL=TEE/BMR).

Habitual physical activity: Habitual physical activity consisted of both non-exercise physical activity (NEPA) and EPA measured over the 14-d period of assessment. The LifeCorder (Suzuken Co., Japan) is a uni-dimensional $\mathrm{AC}$ in a lightweight microcomputer $(6.2 \times 4.6 \times$ $2.6 \mathrm{~cm} ; 42 \mathrm{~g}$ ) designed to detect steps and acceleration rates along the vertical axis at waist level. Prior to use, the AC was programmed with the age, gender, height, and body weight of each subject. Participants were instructed to firmly clip the device to a belt on the right side of their waist and wear it at all times while awake, except when bathing. According to the instructions of the manufacturer, the device was programmed to register and classify data from concurrent 4-s blocks and to analyze this information every $2 \mathrm{~min}$. NEPA was classified as 0 to 2 steps within a 4-s period while EPA involved 3 or more steps being taken in the same timeframe. EPA was categorized as low-, moderate-, and high-intensity, as experimentally defined by the manufacturer. The accumulated duration (time expressed in minutes) of each category of EPA was calculated after removing that of the NEPA, and expressed as the mean values over the 14-d assessment period.

Physical fitness: To assess physical fitness, $\dot{\mathrm{V}} \mathrm{O}_{2} \max$ was determined using an incremental exercise test on a treadmill until volitional exhaustion. Participants started the test at a comfortable walking speed and the workload was then raised by increasing the treadmill speed in increments of $20 \mathrm{~m} / \mathrm{min}$ every $2 \mathrm{~min}$. The incline of the treadmill was maintained at $0 \%$, and both $\dot{\mathrm{V}} \mathrm{O}_{2}$ and HR were constantly monitored. $\dot{\mathrm{V}} \mathrm{O}_{2}$ measurement was assessed using a clip nose and mouthpiece connected to a respiratory valve, with expired air being simultaneously analyzed by an ARCO-1000 respiratory mass spectrometer. HR was recorded minute-by-minute by an "Accurex plus" cardio-frequency meter (Polar electro, Kempele, Finland). $\dot{\mathrm{V}} \mathrm{O}_{2}$ max was determined by extrapolating the relationship between the observed $\dot{\mathrm{V}} \mathrm{O}_{2}$ and $\mathrm{HR}$.

Statistical analyses. All results are expressed as mean \pm SD. Pearson product-moment correlations were calculated to determine the degree of association between any pairs of variables. In this study, we selected ten variables: age, height, body mass index, FM, FFM, percentage body fat, $\dot{\mathrm{V}} \mathrm{O}_{2}$ max and accumulated duration spent for low-, moderate-, and high-intensity EPA that was assumed to be affecting TEE. Body weight (BW) was excluded for multiple stepwise regression analyses because fat-free mass (FFM) and fat-mass (FM) are functions of BW. Then, we entered these variables into multiple stepwise regression analyses (forward selection) to predict TEE in order to examine the extent to which these variables may affect TEE. $p<0.05$ was taken to indicate a statistical significance. All statistical analyses were performed using the statistical package StatView 5.01 (SAS Institute Inc., Cary, NC, USA, 2000-2001).

\section{RESULTS}

The participants' weight remained stable over the $14 \mathrm{~d}$ of the assessment period (initial body weight= $64.9 \pm 10.0 \mathrm{~kg}$ and final body weight $=65.1 \pm 10.1 \mathrm{~kg}$ ). Mean individual body weight change was $-0.2 \pm 0.7 \mathrm{~kg}$ (range, -0.9 to $1.8 \mathrm{~kg}$ ). Pooled data for anthropometrics and energy expenditure are presented in Table 1. Total accumulated duration of NEPA and EPA were $587 \pm 124$ and $93 \pm 29 \mathrm{~min}$, whereas those of low-,

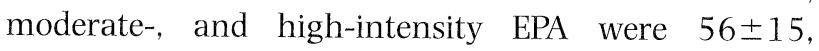
$31 \pm 14$, and $6 \pm 8 \mathrm{~min}$, respectively.

Pearson product-moment correlations demonstrated that, while the mean value of BMR $(r=-0.37, p<0.05)$ declined significantly with age, PAEE and TEE did not (Fig. 1). There were no significant relationships between TEE and low-, moderate- and high-intensity EPA duration, respectively ( $r=0.16,0.08$, and 0.28 , ns), or between PAL and low-, moderate-, and high-intensity EPA duration, respectively $(r=0.21,-0.03$, and $0.28, \mathrm{~ns})$.

Results from a stepwise regression analysis of TEE measured with DLW method with 10 variables are shown in Table 2. The mathematical relation resulting from the stepwise regression that predicts TEE is shown in Eq. (1):

$$
\begin{aligned}
& \text { TEE }(\mathrm{kcal} / \mathrm{d})=\{28.6 \times \text { FFM }(\mathrm{kg})\} \\
& \quad+\{11.2 \times \text { High-intensity EPA }(\min )\}+1114,
\end{aligned}
$$

with a standard error of $\pm 263 \mathrm{kcal} / \mathrm{d}$. The regression analysis indicates that FFM and high-intensity EPA contribute to $51 \%$ of the variability in TEE.

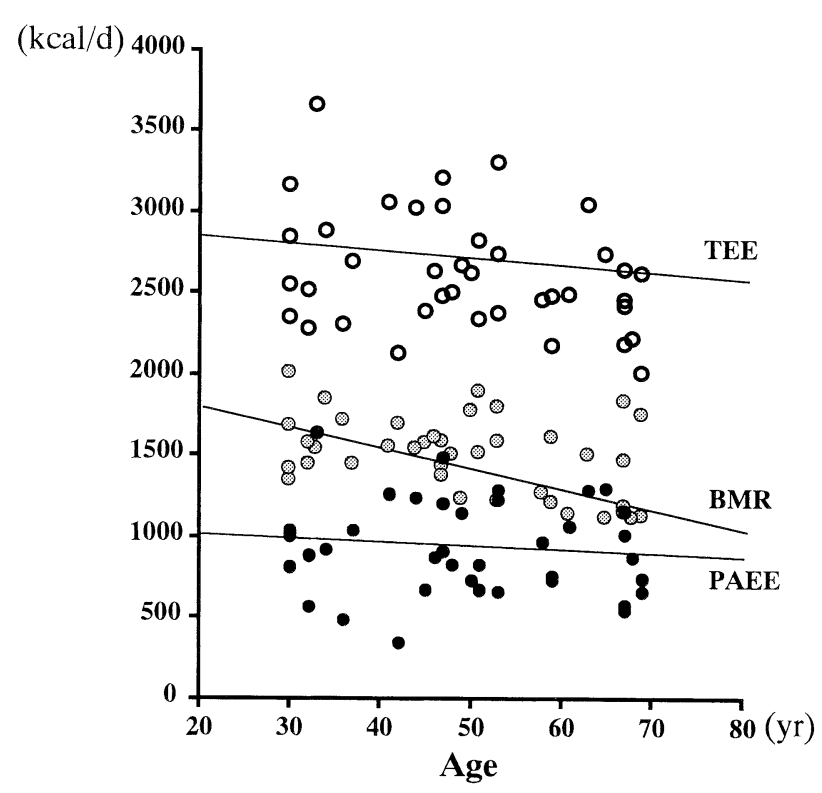

Fig. 1. Relationships between total energy expenditure (TEE, $\bigcirc)$, basal Metabolic rate (BMR, ○) $(p<0.0195)$, and physical activity-induced energy expenditure (PAEE, - ) ( $y$ axios) and age ( $x$ axis). 
Table 2. Multiple stepwise regression analysis relating total energy expenditure (TEE) measured with the use of doubly labeled water to fat-free mass (FFM) and high-intensity EPA $(n=40)$.

\begin{tabular}{|c|c|c|c|c|}
\hline $\begin{array}{l}\text { Dependent } \\
\text { variable }\end{array}$ & Step & $\begin{array}{l}\text { Independent } \\
\text { variable }\end{array}$ & $R^{2}$ & $p$ \\
\hline TEE & 1 & FFM & 0.451 & $<0.001$ \\
\hline TEE & 2 & $\begin{array}{c}\text { high-intensity } \\
\text { EPA }\end{array}$ & 0.513 & $<0.001$ \\
\hline
\end{tabular}

$R^{2}$ is the fraction of explained variance. The last step provides the cumlative $R^{2}$.

\section{DISCUSSION}

In this study we found that high-intensity EPA was identified as an important modulator of TEE in addition to the effect of FFM in healthy working Japanese men 30 to $69 \mathrm{y}$ of age. This may be explained by the contribution of exercise on PAEE. PAEE has been reported as being the most variable component of daily TEE (17, 18), and the most significant indicator of variation between individuals (19). Our findings indicate that participation in high-intensity EPA, even for an accumulated duration of less than half an hour, appears to play an important role for increasing PAEE and concomitantly TEE. Participation in high-intensity EPA presumably also increases both low- and moderate-intensity exercise (due to pre- and post-exercise warming up and cooling down), which may lead to an enhancement of PAEE and TEE.

Since FFM is significantly related to muscle mass, it can be presumed that a high FFM will not only increase BMR, but also may effect PAEE due to the greater percentage of metabolically active tissue (20). Indeed, Cunningham reported that body composition (FFM) is the principal determinant of BMR. Furthermore, the factor of age may rather influence the body composition of an individual (21).

Using whole room indirect calorimetry, Melanson et al. recently demonstrated that difference of exercise intensity at $40 \%$ or $70 \% \dot{\mathrm{V}} \mathrm{O}_{2}$ max did not affect TEE because equal energy costs were loaded on both training level (8). This may indicate that both exercise intensity and duration coincide with affecting TEE. Recently, Westerterp (5) using middle-aged and non-obese subjects of both sexes classified in an active group $(\mathrm{PAL}=1.5$ to 2.1$)$, reported that time spent on moderate-intensity activity has a strong impact on PAL. In contrast, we found no such relationship between PAL and any EPA durations. The reason(s) was not clear, but there were some differences between the two studies in the subjects (ages, sexes, and occupations) and classifications of EPA deduced by the accelerometers. However, further study is required in these areas.

To test the validity of the developed equation, we predict TEE from the 6th revision of the recommended dietary allowances for Japanese (22), in which the average body weight at 30 to 49 and 50 to $69 \mathrm{y}$-old are
67.0 and $62.5 \mathrm{~kg}$, respectively. If body fat is considered at $25 \%$, predicted TEE without high-intensity EPA might be identical with physical activity level III of the recommended energy allowances (REA) on the same age group for 2,550 and $2,300 \mathrm{kcal} / \mathrm{d}$. When high-intensity EPA of 10 to $20 \mathrm{~min}$ is included in the equation, predicted TEE might be kept within in physical activity level IV of the REA for 2,850 and $2,550 \mathrm{kcal} / \mathrm{d}$. Therefore, daily high-intensity EPA might contribute to the increase in TEE, and consequently play a role in the beneficial effects of energy balance (23).

In summary, our study supports the positive association between the total accumulated duration of high-intensity EPA and TEE in healthy Japanese men 30 to $69 \mathrm{y}$ of age. Intensity and duration of the EPA seemed to be significant in determining TEE. Body composition was found to have a greater direct impact on TEE than physical fitness did in this population.

\section{Acknowledgments}

We gratefully acknowledge all participants for enabling this project. We especially appreciate the support of the Japanese Ministry of Health, Labour, and Welfare, Medical Frontier, Strategy Research (H11-KENKOU018) for funding this study.

\section{REFERENCES}

1) Morris JN, Kagan A, Pattison DC, Gardner MJ. 1966. Incidence and prediction of ischemic heart disease in London busmen. Lancet 2(7463): 553-559.

2) American College of Sports Medicine. 1998. ACSM position stand on exercise and physical activity for older adults. Med Sci Sports Exerc 30: 992-1008.

3) Van Etten Ludo MLA, Westerterp KR, Verstappen FTJ, Boon BJB, Saris WHM. 1997. Effect of an 18-wk weight-training program on energy expenditure and physical activity. J Appl Physiol 82(1): 198-304.

4) King GA, Fitzhugh EC, Bassett DR Jr, McLaughlin JE, Strath SJ, Swartz AM, Thompson DL. 2001. Relationship of leisure-time physical activity and occupational activity to the prevalence of obesity. Int J Obes 25: 606612.

5) Westerterp KR. 2001. Pattern and intensity of physical activity. Nature 410: 539.

6) Melanson EL Jr, Freedson PS. 1996. Physical activity assessment: A review of methods. Crit Rev Food Sci Nutr 36(5): 385-396.

7) Schutz Y, Weinsier RL, Hunter GR. 2001. Assessment of free-living physical activity in humans: an overview of currently available and proposed new measures. Obes Res 9: 368-379.

8) Melanson EL, Sharp TA, Seagle HM, Horton TJ, Donahoo WT, Grunwald GK, Hamilton JT, Hill JO. 2002. Effect of exercise intensity on 24-h energy expenditure and nutrient oxidation. J Appl Physiol 92: 1045-1052.

9) Hunter GR, Weinsier RL, Bamman MM, Larson DE. 1998. A role for high intensity exercise on energy balance and weight control. Int J Obes 22: 489-493.

10) Schoeller DA, Van Santen E. 1982. Measurement of energy expenditure in humans by doubly labeled water method. J Appl Physiol 53(4): 955-959.

11) Ebine N, Shimada M, Tanaka H, Nishimuta M, 
Yoshitake Y, Saitoh S, Jones PJH. 2002. Comparative study of total energy expenditure in Japanese men using doubly labeled water method against activity record, heart rate monitoring, and accelerometer methods. Jpn J Phys Fitness Sport Med 51: 151-164 (in Japanese with English summary).

12) Rafamantanantsoa HH, Ebine N, Yoshioka M, Higuchi H, Yoshitake Y, Tanaka H, Saitoh S, Jones PJH. Validation of three alternative methods to measure total energy expenditure against the doubly labeled water method for older Japanese men. J Nutr Sci Vitaminol $\mathbf{4 8}$ : 517-523.

13) Brozek J, Grande F, Anderson JT, Keys A. 1963. Densitometry analysis of body composition: 9th revision of some quantitative assumptions. Ann NY Acad Sci 110: 113-140.

14) Weir JB. 1949. New methods for calculating metabolic rate with special reference to protein metabolism. I Physiol 109: 1-9.

15) Pulfrey SM, Jones PJH. 1996. Energy expenditure and requirement while climbing above $6,000 \mathrm{~m}$. J Appl Physiol 72: 23-28.

16) Ebine N, Feng JY, Homma M, Saitoh S, Jones PJH. 2000. Total energy expenditure of elite synchronized swimmers measured by the doubly labeled water method.
Eur J Appl Physiol 83: 1-6.

17) Pannemans DLE, Westerterp KR. 1995. Energy expenditure, physical activity and basal metabolic rate of elderly subjects. Br J Nutr 73: 571-581.

18) Fuller NJ, Sawyer MB, Coward WA, Paxton PA, Elia M. 1996. Components of total energy expenditure in freeliving elderly men (over 75 years of age): measurement, predict-ability and relationship to quality-of-life indices. Br J Nutr 75: 161-173.

19) Westerterp KR. 2000. Daily physical activity, aging, and body composition. J Nutr Health Aging 4(4): 239242.

20) Poehlman ET. 1992. Energy expenditure and requirements in aging humans. J Nutr 122: 2057-2065.

21) Cunningham JJ. 1980. A reanalysis of the factors influencing basal metabolic rate in normal adults. Am J Clin Nutr 33: 2372-2374.

22) Health Promotion and Nutrition Division, Health Service Bureau, Ministry of Health and Welfare. 1996. Recommended Dietary Allowances for the Japanese, 6th revision, Dai-ichi Shuppan, Tokyo.

23) Brochu M, Starling RD, Ades PA, Poehlman ET. 1999. Are aerobically fit older individuals more physically active in their free-living time? A doubly labeled water approach. J Clin Endocrinol Metab 84: 3872-3876. 